\title{
Difference between actual vs. pathology prostate weight in TURP and radical robotic-assisted prostatectomy specimen
}

\author{
Szilveszter Lukacs ${ }^{1}$, Justin Vale ${ }^{1}$, Evangelos Mazaris ${ }^{1}$ \\ ${ }^{1}$ St Mary's Hospital Imperial College Healthcare NHS Trust, Praed Street, London, W2 1NY, United \\ Kingdom
}

\section{ABSTRACT}

Introduction: To investigate and highlight the effect of formaldehyde induced weight reduction in transurethral resection of prostate (TURP) and radical robotically-assisted prostatectomy (RALP) specimen as a result of standard chemical fixation.

Materials and Methods: 51 patients were recruited from January 2013 to June 2013 who either underwent a TURP $(n=26)$ or RALP $(n=25)$. Data was collected prospectively by the operating surgeon who measured the native, unfixed histology specimen directly after operation. The specimens were fixed in 10\% Formaldehyde Solution BP and sent to the pathology laboratory where after sufficient fixation period was re-weighed. Results: Overall mean age 64.78 years, TURP mean age 68.31 years RALP mean age 61.12years. We found that the overall prostatic specimen $(n=51)$ weight loss after fixation was a mean of $11.20 \%$ (3.78 grams) ( $p \leq 0.0001)$. Subgroup analysis of the native TURP chips mean weight was 16.15 grams and formalin treated mean weight was 14.00 grams $(\mathrm{p} \leq 0.0001)$. Therefore, TURP chips had a mean of $13.32 \%$ (2.15 grams) weight loss during chemical fixation. RALP subgroup unfixed specimen mean weight was 52.08 grams and formalin treated mean weight was 42.60 grams $(p \leq 0.0001)$, a $19.32 \%$ (9.48grams) mean weight reduction.

Conclusion: It has not been known that prostatic chips and whole human radical prostatectomy specimen undergo a significant weight reduction. The practical significance of the accurate prostate weight in patient management may be limited, however, it is agreed that this should be recorded correctly, as data is potential interest for research purposes and vital for precise documentation.

\section{ARTICLE INFO}

\section{Key words:}

Prostatectomy; Transurethral Resection of Prostate; Pathology; Prostate; Robotics; Prostatectomy

Int Braz J Urol. 2014; 40: 823-7

Submitted for publication: January 07, 2014

Accepted after revision:

May 13, 2014

\section{INTRODUCTION}

Formalin is the most commonly used chemical for tissue fixation worldwide as it provides excellent morphological preservation for routine histology (1). Formaldehyde fixation preserves tissue from degradation, and maintains the structure of the cell and of sub-cellular components such as cell organelles (e.g., nucleus, endoplasmic reticulum, mitochondria). Mainly 10\% neutral buffered formalin (4\% formaldehyde) is being used for light microscopy. (1).
Its chemical action in order to preserve tissue is exerted mainly by irreversibly cross-linking protein. The main action of these formaldehyde fixatives is to cross-link amino groups in proteins through the formation of methylene bridges $\left(-\mathrm{CH}_{2}-\right)$, in the case of formaldehyde, or by a $\mathrm{C} 5 \mathrm{H} 10$ cross-links in the case of glutaraldehyde. Formaldehyde will cause a significant amount of prostate tissue shrinkage by 4.1-4.5\%, however we cannot find any scientifically valid result available regarding weight loss resulting by chemical fixation in prostatic specimens $(2,3)$. 
Previous studies were conducted in animal organs (4) to estimate the effect of storage in formalin on organ weights; however, we can not find comparative papers for human prostate even after an extensive literature search.

Measurement of prostate weight can be determined by transabdominal (TAUS) or transrectal ultrasound (TRUS) (5), however increasingly magnetic resonance imaging (MRI) $(6,7)$ and multiparametric MRI are used mainly in oncology setting to obtain more precise and accurate estimation of prostate size for treatment plan. In ter$\mathrm{ms}$ of the prostate size/volume a large 67 TURP (transurethral resection of the prostate) series was analyzed by Mayer et al. (8) which showed a mean pre-operative prostate volume of 47.6 grams, with mean resected prostate tissue of 25.8 grams. In a single center prospective study published by $\mathrm{Ba}-$ dani et al (9), 2766 RALP (robotic-assisted laparoscopic prostatectomy) results showed a mean prostate weight of 49.91 grams (13-220 grams); preoperative weight was not recorded. Generally none of these or other trials recorded when and how is prostatic specimen weight recorded, which could carry a significant bias due to chemical fixation induced weight loss.

Our aim was to quantitatively evaluate the discrepancies in actual versus post formaldehyde-fixed prostate weight in our TURP and RALP specimens involving the influence of formalin fixation.

\section{MATERIAL AND METHODS}

Fifty-one patients were recruited prospectively between January 2013 to June 2013 who either underwent a TURP (standard monopolar resection) $(\mathrm{n}=26)$ for bladder outflow obstruction (B00) or a RALP $(n=25)$ for histologically proven organ confined prostate cancer in our institution. RALP specimens were measured en block with the seminal vesicles. Data was collected for initial measurement of the native, unfixed histology specimen directly after the operation by the operating surgeon and measured without delay on a SECA 856 digital medical scale (Graduation Weight: 1 $\mathrm{g}<3 \mathrm{~kg}>2 \mathrm{~g})$. Weight of the specimen was recorded by the research team on an encrypted pen drive with no access but to the research team. Pa- thologists were unaware of the original specimen weight measured by the research team. Specimens were fixed in formaldehyde solution BP $10 \% \mathrm{v} / \mathrm{v}$ (equivalent to $4 \%$ formaldehyde) with sodium chloride BP $0.9 \% \mathrm{w} / \mathrm{v}$. and sent to the pathology laboratory where after sufficient period of fixation were measured on OHAUS EB3 series scale (Graduation Weight: $1 \mathrm{~g}<3 \mathrm{~kg}>1 \mathrm{~g}$ ). Standard 1-2-5-10-100 grams test weights were used to compare accuracy of the scales to avoid bias from measurement. Both scales measured tester's weight precisely and accurately with no difference in terms of weight. Overall and subgroup analysis of the native untreated prostate weights and chemically treated prostate weights were analyzed with paired t-test.

\section{RESULTS}

A total number of 51 patient were recruited, TURP $(n=26)$ and RALP $(n=25)$ over six months (from January 2013 to June 2013). Demographical characteristics of the patients showed an overall patients' mean age of 64.78 ( $\left.S D_{ \pm} 8.43\right)$, TURP patients' mean age of 68.31 $\left(\mathrm{SD}_{ \pm} 9.43\right)$, RALP patients' mean age of 61.12 $(\mathrm{SD} \pm 5.29)$.

Overall native specimen $(n=51)$ mean weight was 33.76 grams $\left(\mathrm{SD}_{ \pm} 22.22\right)$ and formaldehyde treated mean weight was 29.98 grams ( $\left.\mathrm{SD}_{ \pm} 20.56\right)$, a difference which is extremely statistically significant $(\mathrm{p} \leq 0.0001)$, with a standard error of 0.472 (Figure-1). The mean weight reduction after chemical fixation was $11.20 \%$ compared to native prostatic tissue weight.

Subgroup analysis of native TURP chips showed a mean weight of 16.15 grams $\left(\mathrm{SD}_{ \pm} 9.01\right)$ and formaldehyde treated mean weight of 14.00 grams $\left(\mathrm{SD}_{ \pm} 8.76\right)$ a difference which is extremely statistically significant $(\mathrm{p} \leq 0.0001)$, with a standard error of 0.46 , The mean weight reduction after chemical fixation was $13.32 \%$ compared to native TURP chips weight.

RALP native specimens mean weight was 52.08 grams $\left(S_{ \pm} D_{16.09}\right)$ and formaldehyde treated mean weight of 42.60 grams $\left(S D_{ \pm} 15.36\right)$ a difference which is extremely statistically significant $(p \leq 0.0001)$, with a standard error of 0.696 . 
Figure 1 - Mean weight of the native and the post formaldehyde fixed specimen subgroups in grams. (Overall, TURP chips and RALP)

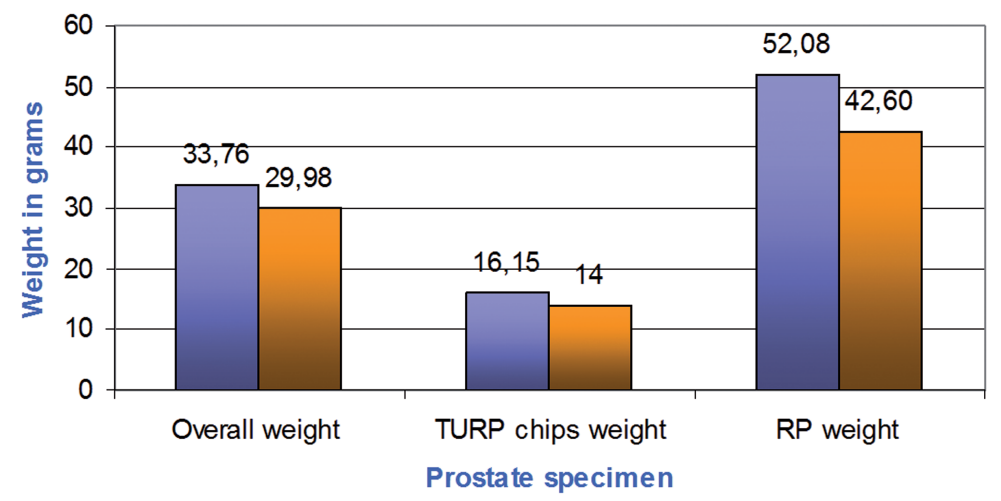

$\square$ Untreated native specimen (grams) $\square$ Formaldehide Treated specimen (grams)

Mean weight reduction of the RALP specimens was $19.32 \%$ after chemical fixation.

Overall final histology mean reporting time was 7.24 days $\left(\mathrm{SD}_{ \pm} 3.43\right)$. TURP specimen final histology mean reporting time was 6.15 days $\left(\mathrm{SD}_{ \pm}+3.86\right)$ and 8.36 days $\left(\mathrm{SD}_{ \pm} 2.51\right)$ for the RALP specimen which was slightly longer (reporting days include weekends as well).

None of the TURP chips contained incidental adenocarcinoma on the final histology report, and all were reported as a benign prostatic hyperplasia (BPH) or adenofibromyomatous hyperplasia.

\section{DISCUSSION}

A large 67 TURP series was analyzed by Mayer et al. (8) which showed a mean pre-operative prostate volume of 47.6 grams, mean resected prostate tissue 25.8 grams with a resection time of 38.5 minutes. In a single center prospective study published by Badani et al (9) 2766 RALP results showed a mean prostate weight of 49.91grams (13-220 grams), with a mean operative time of 154 minutes, preoperative prostate volume was not included. Our data correlates well in terms of the average prostate volume/weight around 45-50 grams in these studies, however none of these studies nor other articles specify precisely how and when the specimen was weighed.

In a common clinical setting, transabdominal (TAUS) or transrectal ultrasound (TRUS) (10) can be used to assess prostate size. There have been studies working on the correlation of TAUS and TRUS in the prostate volume measurement $(5,11)$ which suggest a good correlation between transabdominal estimation of prostatic volume with the transrectal method. Other studies showed (12) that prostatic weight and volume measured with TAUS are overestimated in about $50 \%$ of cases, therefore suggested that TRUS shall remain the gold standard to monitor prostate volume and weight. With the evolution of imaging technology, magnetic resonance imaging (MRI) $(6,7)$ and multiparametric MRI and MR- positron emission tomography (PET) that offer detailed images of the prostate could potentially offer more precise and accurate estimation of prostate size.

Accurate reporting of radical prostatectomy specimens is becoming more important as we gain insights into how cancer therapy should be tailored according to risk categories, therefore handling of these specimens must be standardized, enabling the correct identification of histopathological risk factors for poor outcome (13). When tumor and or prostatic tissue volume is measured planimetrically, results are multiplied by a 
correction factor to compensate for tissue shrinkage caused by processing the specimen (3). As previously reported by Jonmarker et al, the tissue shrinkage after fixation with formalin in radical prostatectomy specimen resulted in an average linear shrinkage of $4.5 \%$ corresponding to a volume correction factor of 1.15 (2).

As well as volume/size, the weight of the prostatic specimen has also gained attention lately as Peiguo et al showed a relationship with low median prostate weight $(49 \mathrm{~g})$ resulting in a significantly higher positive margin rate and incidence of extraprostatic extension (14) in a large prospective study of a laparoscopic radical prostatectomy series. In their study it was 1.523 times more likely to have positive margins with small weight prostates. We found that our RALP specimens had 6\% more mean weight loss (19.32\% vs. $13.32 \%)$ than TURP chips.

Limitations of our study are the fact that two different types of scale were used, however both scales measured tester's weight precisely and accurately with no difference in terms of weight. A potential source of bias for our data could be the fact that the RALP prostate specimens were weighed with the seminal vesicles attached, however, they were weighed again by the pathologist exactly the same way. The true weight of the prostate gland can only be determined if the seminal vesicles are detached from the prostate gland before weighing and that was recommended by the International Society of Urological Pathology (ISUP) Consensus Conference (15). They also concluded that $76 \%$ of participants weighed the prostate with the seminal vesicles attached and it was noted that some urologists request the weight of the entire specimen in order to assign a level of difficulty to the surgical procedure, in which case the weights of the prostate and the seminal vesicles could be combined (15).

Therefore we cannot determine the weight loss of the seminal vesicle as a result of formaldehyde fixation, and we cannot predict how that affected our overall RALP specimen results. On the contrary, detaching the seminal vesicle in theatre could potentially carry a hazard of damaging prostatic and seminal vesicle margins resulting in understaging of the disease (16), therefore the au- thor would not recommend it to be performed by the urologist. Further evaluation is needed to determine the formaldehyde induced seminal vesicle weight loss and shrinkage.

\section{CONCLUSIONS}

Chemical fixation performed with formaldehyde causes a considerable amount of tissue shrinkage; however it has not been known that prostatic chips and whole human radical prostatectomy specimens undergo a significant weight reduction as well. The practical significance of the accurate prostate weight in patient management may be limited, however it is agreed by everyone that this should be recorded, as such data are of potential interest for research purposes and vital for precise documentation. Generally, most of the articles quote the histologically reported prostate weight which is biased by chemical fixation at least 10\%-20\% reduction according to our study findings. These facts raise serious concerns about the accuracy of the previous studies dealing with prostatic specimen weight. The urological and pathological community will have to raise the awareness on chemical fixation induced specimen weight loss and have to reach consensus in order to avoid measurement bias and hence precise weighing which will lead to a clear and accurate data.

\section{CONFLICT OF INTEREST}

None declared.

\section{REFERENCES}

1. Frankel A. Formalin fixation in the '-omics' era: a primer for the surgeon-scientist. ANZ J Surg. 2012;82:395-402.

2. Jonmarker S, Valdman A, Lindberg A, Hellström M, Egevad L. Tissue shrinkage after fixation with formalin injection of prostatectomy specimens. Virchows Arch. 2006;449:297301.

3. Schned AR, Wheeler KJ, Hodorowski CA, Heaney JA, Ernstoff MS, Amdur RJ, et al. Tissue-shrinkage correction factor in the calculation of prostate cancer volume. Am J Surg Pathol. 1996;20:1501-6.

4. Fraser KW. Effect of storage in formalin on organ weight of rabbits. New Zealand Journal of Zoology. 1985;2:169-74. 
5. Styles RA, Neal DE, Powell PH. Reproducibility of measurement of prostatic volume by ultrasound. Comparison of transrectal and transabdominal methods. Eur Urol. 1988;14:266-9.

6. Dianat SS, Rancier Ruiz RM, Bonekamp D, Carter HB, Macura KJ. Prostate volumetric assessment by magnetic resonance imaging and transrectal ultrasound: impact of variation in calculated prostate-specific antigen density on patient eligibility for active surveillance program. J Comput Assist Tomogr. 2013;37:589-95.

7. Runge VM. Current technological advances in magnetic resonance with critical impact for clinical diagnosis and therapy. Invest Radiol. 2013;48:869-77.

8. Mayer EK, Kroeze SG, Chopra S, Bottle A, Patel A. Examining the 'gold standard': a comparative critical analysis of three consecutive decades of monopolar transurethral resection of the prostate (TURP) outcomes. BJU Int. 2012;110:1595601.

9. Badani KK, Kaul S, Menon M. Evolution of robotic radical prostatectomy: assessment after 2766 procedures. Cancer. 2007;110:1951-8.

10. Patel U, Rickards D. Handbook of Transrectal Ultrasound and Biopsy of the Prostate. London: Martin Dunitz, 2002. (Recommendation: B, level of evidence: 3 )

11. Huang Foen Chung JW, de Vries SH, Raaijmakers R, Postma R, Bosch JL, van Mastrigt R. Prostate volume ultrasonography: the influence of transabdominal versus transrectal approach, device type and operator. Eur Urol. 2004;46:352-6.
12. Blanc M, Sacrini A, Avogadro A, Gattamorta M, Lazzerini F, Gattoni F, Cretti F. Prostatic volume: suprapubic versus transrectal ultrasonography in the control of benign prostatic hyperplasia. Radiol Med. 1998;95:182-7.

13. Egevad L. Handling of radical prostatectomy specimens. Histopathology. 2012;60:118-24.

14. Chu PG, Lau SK, Weiss LM, Kawachi M, Yoshida J, Ruel C, et al. Assessment of low prostate weight as a determinant of a higher positive margin rate after laparoscopic radical prostatectomy: a prospective pathologic study of 1,500 cases. Surg Endosc. 2009;23:1058-64.

15. Samaratunga H, Montironi R, True L, Epstein JI, Griffiths DF, Humphrey PA. International Society of Urological Pathology (ISUP) Consensus Conference on Handling and Staging of Radical Prostatectomy Specimens. Working group 1: specimen handling. Mod Pathol. 2011;24:6-15.

16. Bong GW, Ritenour CW, Osunkoya AO, Smith MT, Keane TE. Evaluation of modern pathological criteria for positive margins in radical prostatectomy specimens and their use for predicting biochemical recurrence. BJU Int. 2009;103:327-31.

Correspondence address:

Szilveszter Lukacs, MRCS, MD St Mary's Hospital, Imperial College Healthcare NHS Trust Praed Street, City of Westminster, London, W21NY, United Kingdom Telephone: +44020 3312-6666 Email: slylukacs@yahoo.com 